\title{
Long-Range Predissociation in Two-Color Photoassociation of Ultracold Na Atoms
}

\author{
P. A. Molenaar, P. van der Straten, and H. G. M. Heideman \\ Debye Institute, Department of Atomic and Interface Physics, Utrecht University, P.O. Box 80.000, \\ 3508 TA Utrecht, The Netherlands
}

(Received 13 March 1996)

We report two-color photoassociative ionization of sodium in a magneto-optical trap. The experimental results yield information on both singly and doubly excited states. We find that the highest bound vibrational levels $(v>20)$ of the singly excited $0_{g}^{-}$state predissociate into the $3^{2} P_{3 / 2}+3^{2} S_{1 / 2}\left(F_{g}=1\right)$ dissociation continuum due to avoided crossings of the hyperfine components of this potential with other molecular symmetries. Based on symmetry and energy consideration we argue that a doubly excited $1_{u}$ state remains autoionizing even when excited only a few $\mathrm{GHz}$ above the dissociation continuum. [S0031-9007(96)00946-5]

PACS numbers: 33.80.Gj, 32.80.Pj, 33.15.Mt, 34.50.Rk

The rapid development of laser cooling techniques (for a review see [1]) during the last decade has opened the path for study of cold collisions. The narrow thermal energy distribution in which the atoms can be prepared allows spectroscopy with extremely high resolution. One of the reactions that has been studied is the photoassociative ionization reaction for sodium $\left(\mathrm{Na}+\mathrm{Na}+\hbar \omega \rightarrow \mathrm{Na}_{2}{ }^{*}\right.$, followed by $\mathrm{Na}_{2}{ }^{*}+\hbar \omega \rightarrow \mathrm{Na}_{2}{ }^{+}+e^{-}$). The structures in the production of ions as a function of the frequency of the exciting light in these experiments reflects the boundstate structure of the singly excited states $\left(\mathrm{Na}_{2}{ }^{*}\right)$. The second step, which is a bound-free transition, displays no observable structure in these experiments [2-6]. The reaction mechanism at those low energies displays some characteristics different from those in the reaction mechanism at typical thermal temperatures. At thermal energies the associative ionization (AI) reaction involves two atoms prepared in excited states, which due to their thermal motion reach the reaction region where autoionization takes place. At temperatures that can be reached with laser cooling techniques the velocity of the excited atoms is so low (typically a few $\mathrm{cm} / \mathrm{s}$ ) that they propagate only a few $a_{0}$ before they spontaneously emit a photon and decay to the ground state. So the colliding excited atom pair will not survive to the reaction region.

Until now, the reaction at low temperature has been described as a three step process [5]. First, the two colliding atoms are excited at long range to a bound singly excited molecular state (Fig. 1). Second, due to the attraction in this bound intermediate state the atoms are effectively accelerated to each other and reach the short internuclear distances where direct photoionization takes place. To distinguish this reaction from the associative ionization reaction this mechanism is referred to as photoassociative ionization (PAI).

A number of groups has studied this reaction using only a single exciting laser for the two successive excitation steps $[5,6]$. Since the last step in this reaction is insen- sitive to the exciting laser frequency, they found that the production of ions as a function of frequency reflects the structure of the bound singly excited sates. Unfortunately, this excitation scheme is restricted to those singly excited states that allow the reaction partners to reach the small internuclear distances where the second excitation step is possible. Excitation at long range by the red detuned light, required to make the first excitation step, to the relatively flat doubly excited potentials is energetically forbidden. Furthermore, the PAI reaction mechanism yields no information on the doubly excited states leading to AI.

To overcome those problems we use two independent laser frequencies for the two excitation steps instead of one laser frequency for both steps. The atom pair is still excited by the first laser to bound singly excited states but the second excitation step can be controlled independently and is not restricted to excite directly to $\mathrm{Na}_{2}{ }^{+}$. Excitation to free doubly excited states becomes possible if the total energy of the two photons is above the dissociation limit. Excitations to energies a few $\mathrm{GHz}$ above this dissociation limit give sufficient kinetic energy

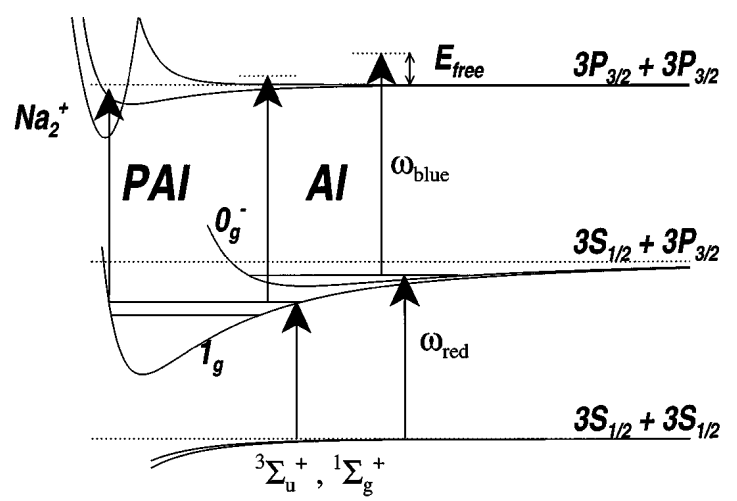

FIG. 1. Model of the two different excitation mechanisms discussed in the text. The PAI mechanism is indicated by the arrow in the close-range region. The two arrows at long range indicate the excitation scheme for the AI reaction. 
for the reaction partners to reach the small internuclear distances before spontaneous emission occurs.

The realization of the two appropriate frequencies from a single laser is by no means a trivial task. Electrooptic (EOM's) and acousto-optic (AOM's) modulators only generate sidebands up to a few $\mathrm{GHz}$ and the alternative solution of using two different exciting lasers requires a lot of resources. We therefore constructed a novel device that generates a sideband at approximately $30 \mathrm{GHz}$. The device consists of a single-mode optical fiber (York, HB600) saturated with $20 \mathrm{~mW}$ input laser power. All the incident light above this threshold is completely reflected back and frequency shifted due to stimulated Brillouin scattering (SBS) in the fiber. The frequency shift of this SBS light is given by $\Delta \omega_{\mathrm{SBS}} / 2 \pi=2 n v_{S} / \lambda$ $[7,8]$. With $n=1.458$ the refractive index of the fiber, $v_{S}=5760 \mathrm{~m} / \mathrm{s}$ the velocity of sound in the fiber, and $\lambda=589.0 \mathrm{~nm}$ the wavelength of the incident light, we find $\Delta \omega_{\mathrm{SBS}} / 2 \pi=28.51 \mathrm{GHz}$. Our experimentally observed value of $28.53(4) \mathrm{GHz}$ lies close to this theoretical prediction. We will refer to the unperturbed probe beam frequency as $\omega_{\text {blue }}$, whereas the redshifted sideband with frequency $\omega_{\text {blue }}-\Delta \omega_{\text {SBS }}$ will be referred to as $\omega_{\text {red }}$. We verified that the spectral distribution of the redshifted light did not differ from the unperturbed probe light by observing the beat signal from the cooling laser and the two probe laser frequencies. We found that all spectral widths are close to the bandwidth of the cooling laser $(\sim 1 \mathrm{MHz})$. A fraction of SBS light is internally reflected on the entrance facet of the fiber $(R \approx 3.4 \%)$ and transmitted in the forward direction. This light together with the unshifted frequency of the unperturbed probe laser light is used in the two-color experiments.

The setup for the magneto-optical trap (MOT) is similar to that found in other laboratories [9]. Three counterpropagating pairs of intersecting, orthogonal laser beams with pairwise $\sigma^{+}-\sigma^{-}$polarization capture slow atoms from a sodium background vapor. The frequency of the beams is detuned $10 \mathrm{MHz}$ to the red of the cooling transition $\omega_{\text {res }}=3^{2} S_{1 / 2}\left(F_{g}=2\right) \rightarrow 3^{2} P_{3 / 2}\left(F_{e}=3\right)$. Optical trapping in the $F_{g}=1$ ground state is prevented by a second, repumping, laser frequency tuned close to the $F_{g}=1 \rightarrow F_{e}=2$ transition. An EOM operating at $860.0 \mathrm{MHz}$ generates both frequencies from the incoming beam with a conversion efficiency of $34 \%$ per beam. The magnetic field gradient of $20 \mathrm{G} / \mathrm{cm}$ is generated by two identical solenoids outside the vacuum. The number of trapped atoms of $5 \times 10^{6}$ at a density of $10^{10} \mathrm{~cm}^{-3}$ is typical for vapor cell MOT's. By switching the MOT beams on and off with an AOM operating at $10 \mathrm{kHz}$ and a duty cycle of $50 \%$ we obtained a cold sample unaffected by the cooling light for periods of $50 \mu \mathrm{s}$. The number of trapped atoms was almost unaffected by this action. The motion of the atoms during this time interval can be neglected given the temperature of the MOT near the Doppler limit of $240 \mu \mathrm{K}$. During the off periods of the cooling beam we illuminated the MOT cloud with light from the second, independently tunable probe laser. This light is focused on the MOT cloud to spot sizes between 30 and $300 \mu \mathrm{m}$. The probe beam is switched off during the cooling periods of the MOT to minimize possible distortion of the cloud when getting close to the atomic resonance frequencies.

The $\mathrm{Na}_{2}{ }^{+}$ions produced in the MOT are measured with a channeltron located $30 \mathrm{~mm}$ from the MOT. The pulses from the channeltron are amplified and divided over two channels. One channel is exclusively opened during the probing periods, with a $12 \mu$ s delay to avoid measuring ions formed during the cooling period. The other channel measures the ion rate during the MOT cooling periods. This serves as a check for MOT stability. All channels were measured simultaneously and recorded in a PC, while scanning the frequency of the probe laser.

Figure 2 shows the observed ion spectrum during the probing phase when $\omega_{\text {blue }}$ and $\omega_{\text {red }}$ are present. Given those two frequencies and the two different reaction channels (AI and PAI) it is not a priori evident which process leads to ionization. However, our measurements make a clear distinction between the options and will demonstrate the preferential path leading to ionization. First we notice that the observed ion signal was exclusively present when we had the two different frequencies incident on the MOT. The reaction mechanism must consequently involve the absorption of both $\omega_{\text {red }}$ and $\omega_{\text {blue }}$. In both proposed ionization mechanisms the second excitation step is a structureless bound-free transition, so we attribute the observed structures to emerge from the singly excited bound states. Thus the observation of regular series of vibrational levels in Fig. 2 directly below the atomic resonance frequency for the red photon $\left(\omega_{\text {red }}<\omega_{\text {res }}\right)$ indicates that the first excitation step is made by $\omega_{\text {red }}$.
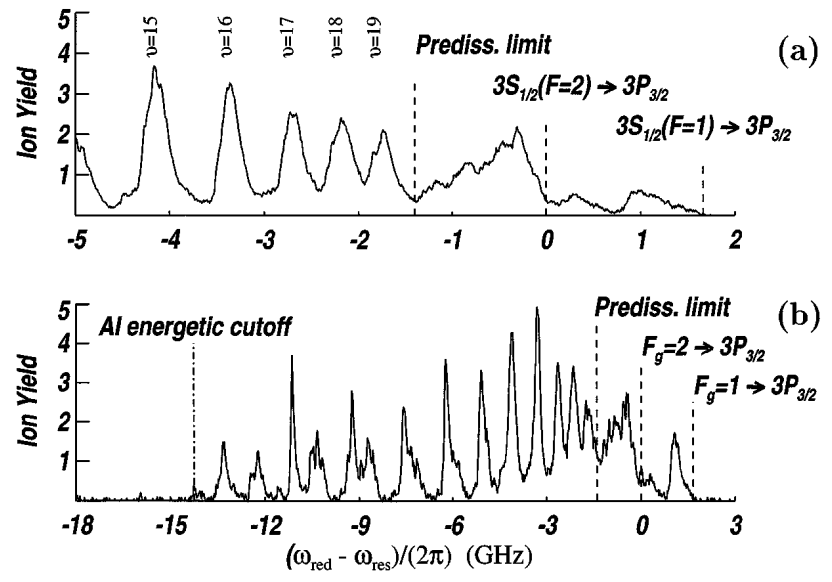

FIG. 2. Ion spectrum as a function of $\omega_{\text {red }}$. (a) gives a more detailed view of the first $5 \mathrm{GHz}$ of (b). The energy threshold for the AI process at $-14.19 \mathrm{GHz}$ can easily be recognized. At small detunings the vibrational series resulting from the $0_{g}^{-}$ displays the predicted cutoff near the predissociation limit of approximately $-1.4 \mathrm{GHz}$. 
Consequently, the second step must be made with the higher energetic photon, $\omega_{\text {blue }}$.

An essential difference between AI and PAI reactions is that the first has an energy threshold where the total energy of the two photons is equal to the dissociation limit for $\mathrm{Na}_{2}{ }^{* *}$. The energy threshold for the PAI ionization mechanism is far out of the range of energies that we observe; the $\mathrm{Na}_{2}{ }^{+}$potential comes down $400 \mathrm{~cm}^{-1}$ below the $3 P-3 P$ dissociation limit. The energetic threshold for the AI mechanism is easily calculated from $\omega_{\text {red }}+\omega_{\text {blue }}=2 \omega_{\text {res }}$ and $\omega_{\text {red }}=\omega_{\text {blue }}-\Delta \omega_{\text {SBS }}$. We therefore only form ions through the AI mechanism if $\omega_{\text {red }}>\omega_{\text {res }}-\Delta \omega_{\text {SBS }} / 2$. The experimentally observed cutoff of the vibrational series at $\left(\omega_{\text {red }}-\omega_{\text {res }}\right) / 2 \pi \approx$ $-14.0 \mathrm{GHz}$ indeed corresponds to the predicted threshold at $\Delta \omega_{\mathrm{SBS}} / 2 \pi=-14.26 \mathrm{GHz}$. So the complete reaction scheme can be summarized as follows:

$$
\begin{aligned}
\mathrm{Na}+\hbar \omega_{\text {red }}+\hbar \omega_{\text {blue }} & \rightarrow \mathrm{Na}^{*}(v, J)+\hbar \omega_{\text {blue }} \\
& \rightarrow \mathrm{Na}^{* *} \rightarrow \mathrm{Na}^{+}+e^{-} .
\end{aligned}
$$

The two vibrational series that can be recognized in Fig. 2 are the singly excited $0_{g}^{-}$and $1_{g}$ states [Hunds case (c) notation]. They are both asymptotically connected to the $3^{2} S_{1 / 2}+3^{2} P_{3 / 2}$ dissociation limit. The narrow peaks result from the $0_{g}^{-}$and the broad ones from the $1_{g}$ state. The identification is based on the same arguments as in [5]. The potential must be of attractive character for large $R$. This restricts the possible states to $0_{u}^{+}$, $0_{g}^{-}, 1_{g}, 1_{u}$, and $2_{u}$ since only these states asymptotically correlate to $3^{2} S_{1 / 2}+3^{2} P_{3 / 2}$ and are attractive. Two of these states are excluded. Excitation to the $2_{u}$ from the $\Sigma$ ground states is dipole forbidden. The depth of $1_{u}$ state is only $5 \mathrm{GHz}$ and would therefore be seen only in the first

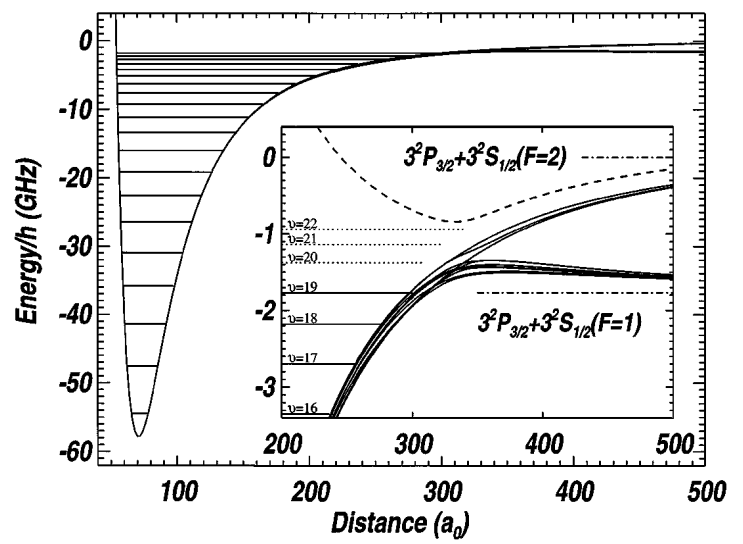

FIG. 3. Calculated potential energies and bound states for the hyperfine components of the $0_{g}^{-}$. The inset shows an enlarged view for small detuning. The bound states have been indicated with the solid lines. The dotted lines indicate the positions of the hypothetical higher bound states. The dashed line indicates one of the potentials that causes the avoided crossings. Most of the $0_{g}^{-}$potentials are connected to $3^{2} S_{1 / 2}\left(F_{g}=1\right)+3^{2} P_{3 / 2}$; only three connect to $3^{2} S_{1 / 2}\left(F_{g}=2\right)+3^{2} P_{3 / 2}$.
$5 \mathrm{GHz}$ whereas our series extends to $14 \mathrm{GHz}$. Finally, we compared the observed width of the peaks with the calculated spread in the energies of the potentials due to hyperfine interaction, which is approximately $0.1 \mathrm{GHz}$ for the $0_{g}^{-}, 0.3 \mathrm{GHz}$ for the $0_{u}^{+}$, and $1 \mathrm{GHz}$ for the $1_{g}$ state. We conclude that we only observe vibrational series of the $0_{g}^{-}$and $1_{g}$ states. The same two series are observed in the trap loss spectra and the $1_{g}$ state is also observed in the single color ionization spectra.

We numerically evaluated the energies of the bound states in the $0_{g}^{-}$potential using the existing analytical expression for this potential from [10]. Although we found convincing agreement between the experimental and calculated results for the lowest bound states, the calculated results for the highest bound states deviated significantly from the experimental results. Those differences could be explained by also taking into account the molecular hyperfine interaction in the calculations. We calculated the adiabatic potential curves for all the 230 nondegenerate hyperfine components asymptotically connected to the $3^{2} P_{1 / 2,3 / 2}+3^{2} S_{1 / 2}$ pair, by using the atomic basis sets and including the experimental asymptotic atomic energies. The interaction was the appropriate dipole-dipole interaction where we used a $C_{3}$ coefficient of 6.210 a.u. [11]. The Hamiltonian was split into six subspaces with different projections of the total angular momentum $\left|m_{F}\right|$ on the internuclear axis. (States with negative $m_{F}$ are degenerate with those with positive $m_{F}$.) After diagonalizing the Hamiltonian we consider the ten nondegenerate components that constitute the $0_{g}^{-}$state (Fig. 3). Seven of these asymptotically connect to $3^{2} P_{3 / 2}+3^{2} S_{1 / 2}\left(F_{g}=1\right)$, not to $3^{2} P_{3 / 2}+3^{2} S_{1 / 2}\left(F_{g}=2\right)$. This is due to

TABLE I. Measured and calculated vibrational energy levels for the $0_{g}^{-}$state. The energies are with respect to the $3^{2} S_{1 / 2}\left(F_{g}=2\right)+3^{2} P_{3 / 2}\left(F_{e}=3\right)$ asymptote. The calculated energies display the weighted average over the ten hyperfine components and the total spread in energy for those different hyperfine components. The $v=8$ signal has been obtained

\begin{tabular}{|c|c|c|c|c|}
\hline \multirow[b]{3}{*}{$v$} & \multicolumn{4}{|c|}{ Energy (GHz) } \\
\hline & \multicolumn{2}{|c|}{ Experimental } & \multicolumn{2}{|c|}{ Calculated } \\
\hline & This Work & Ref. [6] & Average & Spread \\
\hline 19 & $-1.769(22)$ & & -1.741 & 0.196 \\
\hline 18 & $-2.177(13)$ & & -2.171 & 0.192 \\
\hline 17 & $-2.697(12)$ & & -2.706 & 0.183 \\
\hline 16 & $-3.347(16)$ & & -3.360 & 0.165 \\
\hline 15 & $-4.134(13)$ & & -4.154 & 0.143 \\
\hline 14 & $-5.114(12)$ & & -5.115 & 0.121 \\
\hline 13 & $-6.260(10)$ & & -6.267 & 0.101 \\
\hline 12 & $-7.605(19)$ & $-7.5(3)$ & -7.640 & 0.082 \\
\hline 11 & $-9.239(14)$ & $-9.3(3)$ & -9.265 & 0.067 \\
\hline 10 & $-11.127(12)$ & $-11.1(3)$ & -11.176 & 0.055 \\
\hline 9 & $-13.343(16)$ & $-13.5(3)$ & -13.409 & 0.045 \\
\hline 8 & $*-16.020(10)$ & $-16.06(3)$ & -16.000 & 0.036 \\
\hline
\end{tabular}
from excitations from the $F_{g}=1+F_{g}=2$ ground state pair. 
avoided crossings near $R=350 a_{0}$ with states of other symmetries but with equal $m_{F}$. Only the $m_{F}=3$ component and two of the $m_{F}=0$ components connect to $3^{2} S_{1 / 2}\left(F_{g}=2\right)+3^{2} P_{3 / 2}$. We further notice that the behavior of all the hyperfine components for $R$ below $350 a_{0}$ is similar to the analytic expression mentioned above, with an apparent dissociation limit close to $3^{2} P_{1 / 2}\left(F_{g}=2\right)+3^{2} P_{3 / 2}$. We calculated the energies of the bound states for all hyperfine potentials and found excellent agreement between the experimental results and the weighted average of the calculated energies of the bound states (Table I). Also the agreement with the experimental results of Ref. [6] is satisfactory. The highest level that can be sustained in most of the hyperfine potentials is the $v=19$ state. Possible higher bound states predissociate into the $3^{2} S_{1 / 2}\left(F_{g}=1\right)+3^{2} P_{3 / 2}$ dissociation continuum. This unanticipated result is supported by our experimental observations [Fig. 2(a)]; vibrational levels up to $v=19$ can easily be distinguished and the series is abruptly cut off at the predicted predissociation continuum of approximately $1.4 \mathrm{GHz}$ below $\omega_{\text {res }} / 2 \pi$. One should notice that the energy corresponding to the transition $3^{2} S_{1 / 2}\left(F_{g}=2\right)+3^{2} S_{1 / 2}\left(F_{g}=2\right) \rightarrow$ $3^{2} S_{1 / 2}\left(F_{g}=1\right)+3^{2} P_{3 / 2}$ does not correspond to any atomic transition. This process consists of exciting one atom to $3^{2} P_{3 / 2}$ and changing the angular momentum of the other ground state atom from $F_{g}=2$ to $F_{g}=1$. This is an example of a hyperfine changing collision.

Although the associative ionization mechanism is widely studied, the symmetries of the doubly excited states leading to $\mathrm{Na}_{2}{ }^{+}$is still the subject of intense study [12]. Our measurements elucidate these problems, since we prepare the doubly excited states from singly excited molecular states of known symmetries. The set of entrance channels leading to autoionization is therefore limited to those that can be excited from the singly excited potentials.

Excitation from the singly excited $0_{g}^{-}$and $1_{g}$ [Hunds case (c)] states is restricted to the doubly excited states $0_{u}^{-}$and $1_{u}$ (from $0_{g}^{-}$and $1_{g}$ ) and $1_{u}^{+}$and $2_{u}$ (only from $1_{g}$ ). Since the ionization reaction via the singly excited $0_{g}^{-}$state is observed, the doubly excited state $0_{u}^{-}$or $1_{u}$ must be autoionizing. These autoionizing channels can also be excited from the singly excited $1_{g}$ state. In the inner region those two doubly excited states adiabatically connect to Hunds case (a) states $8^{3} \Sigma_{u}^{+}$(connected to a $1_{u}$ and $0_{u}^{-}$state), $1^{1} \Sigma_{u}^{-}\left(0_{u}^{-}\right)$, and $2^{3} \Delta_{u}\left(1_{u}\right)$. Autoionization of the $1^{1} \Sigma_{u}^{-}$state to the lowest ionic state $\left(X^{2} \Sigma_{g}^{+}\right)$, however, is very unlikely due to symmetry restrictions. So only three possible autoionizing channels remain: $1_{u}\left(2^{3} \Delta_{u}\right), 1_{u}\left(8^{3} \Sigma_{u}^{+}\right)$, and $0_{u}^{-}\left(8^{3} \Sigma_{u}^{+}\right)$.

A further restriction on the number of channels leading to AI is based on energy arguments. The preparation of the doubly excited states from the singly excited purely long-range $0_{g}^{-}$state must occur at internuclear separations larger than $55 a_{0}$. Since the frequency of the second exciting photon and energy of the singly excited state are known, the total energy in the doubly excited state can be determined and lies between 1.7 (by excitation from $v=9)$ and $24.8 \mathrm{GHz}(v=19)$ above the $3^{2} P_{3 / 2}+3^{2} P_{3 / 2}$ dissociation limit. So only those doubly excited states that contain no barriers larger than a few $\mathrm{GHz}$ in the range between $55 a_{0}$ and $6 a_{0}$ (where autoionization occurs) can lead to associative ionization. For the previously mentioned three states only the $1_{u}$ (adiabatically connected to $2^{3} \Delta_{u}$ ) satisfies this criterion. The $1_{u}$ and $0_{u}^{-}$states adiabatically connected to $8^{3} \Sigma_{u}^{+}$ are repulsive at long range. So we have demonstrated, independently of the detailed behavior of the potentials at small internuclear separations, that at energies only a few $\mathrm{GHz}$ above the dissociation limit the long-range entrance channel $1_{u}$ (adiabatically connected to $2^{3} \Delta_{u}$ ) leads to autoionization at short range.

In summary, we have demonstrated associative ionization of sodium at kinetic energies of a few $\mathrm{GHz}$ using two-color photoassociative spectroscopy. Based on energy and symmetry arguments our experiments provide stringent restrictions on the channels that lead to associative ionization at these energies. We find that the $1_{u}$ (adiabatically connected to $2^{3} \Delta_{u}$ ) entrance channel must remain autoionizing at low energies. We furthermore found that the purely long-range, singly excited $0_{g}^{-}$state can only sustain 20 bound states. Possible higher bound states predissociate near $R=350 a_{0}$ into a $3^{2} S_{1 / 2}\left(F_{g}=1\right)$ and a $3^{2} P_{3 / 2}$ atom, although the apparent dissociation limit lies at the $3^{2} S_{1 / 2}\left(F_{g}=2\right)+3^{2} P_{3 / 2}$ dissociation energy. This predissociation is due to avoided crossings of the hyperfine components of the $0_{g}^{-}$state with the hyperfine components of other molecular states.

[1] H. Metcalf and P. van der Straten, Phys. Rep. 4 \& 5, 204 (1994).

[2] V. Bagnato, L. Marcassa, C. Tsao, Y. Wang, and J. Weiner, Phys. Rev. Lett. 70, 3225 (1993).

[3] M. E. Wagshul et al., Phys. Rev. Lett. 70, 2074 (1993).

[4] V. Bagnato et al., Phys. Rev. A 48, 2523 (1993).

[5] P. D. Lett et al., Phys. Rev. Lett. 71, 2200 (1993).

[6] L. P. Ratcliff, M. E. Wagshul, P. D. Lett, S. L. Rolston, and W. D. Phillips, J. Chem. Phys. 101, 2638 (1994).

[7] Y. Aoki, K. Tajima, and I. Mito, J. Lightwave Technol. 6, 710 (1988).

[8] Y. Aoki and K. Tajima, J. Opt. Soc. Am. B 5, 358 (1988).

[9] C. Monroe, W. Swann, H. Robinson, and C. Wieman, Phys. Rev. Lett. 65, 1571 (1990).

[10] M. Movre and G. Pichler, J. Phys. B 10, 2631 (1977).

[11] U. Volz et al., Phys. Rev. Lett. 76, 2862 (1996).

[12] O. Dulieu, S. Magnier, and F. Masnou-Seeuws, Z. Phys. D 32, 229 (1994). 